\title{
Prefrontocerebellar transcranial direct current stimulation increases amplitude and decreases latency of P3b component in patients with euthymic bipolar disorder
}

\author{
This article was published in the following Dove Press journal: \\ Neuropsychiatric Disease and Treatment \\ 19 November 2015 \\ Number of times this article has been viewed
}

Francesco Saverio Bersani

Amedeo Minichino

Francesco Fattapposta

Laura Bernabei

Francesco Spagnoli

Daniela Mannarelli

Marta Francesconi

Caterina Pauletti

Alessandra Corrado

Lucilla Vergnani

Ines Taddei

Massimo Biondi

Roberto Delle Chiaie

Department of Neurology and Psychiatry, Sapienza University of

Rome, Rome, Italy
Correspondence: Amedeo Minichino Department of Neurology and Psychiatry, Sapienza University of Rome, Viale dell'Università 30, Rome, Italy Email amedeomin@gmail.com
Introduction: Neurocognitive impairments have been observed in patients with bipolar disorder (BD) even during the euthymic phase of the disease, potentially representing traitassociated rather than state-associated characteristics of the disorder. In the present study, we used transcranial direct current stimulation (tDCS) applied to cerebellar and prefrontal cortices to improve the neurophysiological performances of patients with euthymic BD.

Methods: Twenty-five outpatients with BD underwent open-label prefrontocerebellar tDCS for 3 consecutive weeks. Neurophysiological performances were assessed through the examination of the P3b and P3a subcomponents of P300 event-related potential at baseline and after stimulation.

Results: Compared to baseline, P3b component after tDCS showed significantly higher amplitude and shorter latency (latency: $\mathrm{Fz} P=0.02, \mathrm{Cz} P=0.03$, and $\mathrm{Pz} P=0.04$; amplitude: $\mathrm{Fz} P=0.24$, $\mathrm{Cz} P=0.02$, and $\mathrm{Pz} P=0.35)$.

Conclusion: In our sample of patients with euthymic BD, concomitant prefrontoexcitatory and cerebellar-inhibitory modulations led to improved brain information processing stream. This improvement may at least partially result from neuroplastic modulation of prefrontocerebellar circuitry activity.

Keywords: mood disorders, tDCS, cerebellum, P300, dorsolateral prefrontal cortex, eventrelated potentials

\section{Introduction}

Bipolar disorder (BD) is a severe and disabling disease. The course of BD has traditionally been viewed as episodic, that is with symptomatic and functional recovery between mood episodes. ${ }^{1}$ This view has recently been challenged by evidence documenting how, despite symptomatic improvements or recovery following mood episodes, many individuals with $\mathrm{BD}$ experience difficulties in daily functioning (eg, there are higher rates of unemployment and disability among individuals with BD than in the normal population). ${ }^{2-7}$

Cognitive and functional impairments have been observed in patients with BD even during the euthymic phase of the disease, potentially representing trait-associated rather than state-associated characteristics of the disorder. ${ }^{2-7}$ From a neurophysiological point of view, event-related potentials (ERPs) allow the identification of specific neurocognitive deficiencies. ${ }^{8-10}$ In particular, the P300 component has been studied widely, and it is believed to be related to stimuli categorization as an indicator of selective attention and memory updating. ${ }^{8}$ The P300 consists of two main subcomponents, namely $\mathrm{P} 3 \mathrm{a}$ and $\mathrm{P} 3 \mathrm{~b} .{ }^{8,10}$ The $\mathrm{P} 3 \mathrm{a}$ is elicited by a distracter stimulus, and it has been 
interpreted as a neural correlate of the orienting response; the $\mathrm{P} 3 \mathrm{~b}$ component, which is elicited by a target rare stimulus, reflects neuronal activity associated with revision of the mental representation of the previous event within the stimulus environment. ${ }^{8}$

Recent studies hypothesized that trait-associated neurocognitive impairments in patients with $\mathrm{BD}$ could be related to prefrontocerebellum circuitry dysfunctions and, in particular, to the loss of the physiological inverse metabolic activity between dorsolateral prefrontal cortex (hypoactivity) and subcortical areas, such as thalamus and cerebellum (hyperactivity). ${ }^{11-16}$ Therefore, the aim of this preliminary study was to improve the brain information processing stream (assessed through P300 ERP) stimulating cerebellar and prefrontal cortices with transcranial direct current stimulation (tDCS), a form of neuromodulation that uses constant, low current delivered to the brain area of interest via inhibitory (cathodal) and excitatory (anodal) electrodes. ${ }^{17,18}$

\section{Methods}

\section{Participants and study design}

The sample consisted of 27 outpatients (ten males and 17 females; mean age: $45.9 \pm 12.8$ ) with a diagnosis of $\mathrm{BD}$ type I $(n=16)$ or II $(n=11)$ in the euthymic phase of the disease defined by Hamilton Depression Rating Scale $<7$ and Young Mania Rating Scale $<7$ (disease duration: 18.01 11.09 years). Diagnoses were made through the Structured Clinical Interview for Diagnostic and Statistical Manual of Mental Disorders fourth edition Axis I disorders. ${ }^{19,20}$ Patients were consecutively recruited at the Policlinico Umberto I University Hospital of Rome. Patients were screened and excluded for having significant concomitant neurological or organic diseases, comorbid Axis I diagnoses, left handedness, pharmacological treatment with typical antipsychotics, or hospitalization in the last 12 months. All patients were on stable pharmacological treatment with lithium $(n=13)$, anticonvulsants $(n=18)$, atypical antipsychotics $(n=18)$, benzodiazepines $(n=11)$, and/or antidepressants $(n=6)$ for at least 2 months. The tDCS was applied to patients in addition to the standard pharmacological maintenance therapies, which remained unchanged along the intervention. These participants had previously been enrolled in our research in other studies with different aims and objectives. ${ }^{13,14,21}$ The research protocol was approved by the institutional review board of Policlinico Umberto I University Hospital and Sapienza University of Rome, in accordance with the Declaration of Helsinki, 1975. All patients signed a written informed consent before the tDCS treatment.

\section{Transcranial direct current stimulation}

The tDCS is a brain modulation technique using constant, low current delivered directly to the brain area of interest via inhibitory (cathodal) and excitatory (anodal) electrodes. ${ }^{17,18}$ In our study, tDCS electrode montage was as follows: cathodal tDCS on the right cerebellar cortex, $1 \mathrm{~cm}$ below and $4 \mathrm{~cm}$ lateral to the inion, and anodal tDCS over the left dorsolateral prefrontal cortex (ie, at Fp1 according to the International 10/20 System for EEG electrodes). The intensity of stimulation was set at $2 \mathrm{~mA}$ and delivered for 20 minutes every working day (Monday to Friday) for 3 consecutive weeks. More details can be found in previously published articles. ${ }^{13,14}$

\section{Neurophysiological evaluation}

Neurophysiological evaluation consisted of an ERP evaluation with a P300 Novelty Task. The electroencephalogram signals were recorded with F3, Fz, F4, C3, Cz, C4, P3, Pz, and P4 scalp electrodes, according to the International 10-20 System. The P300 Novelty Task consisted of a sequence of three tones (standard, target, and novel). The standard stimulus was 1,000 Hz tone (duration: $200 \mathrm{~ms}$; rise-fall times: $10 \mathrm{~ms}$; intensity: $80 \mathrm{~dB}$ sound pressure level (SPL); and probability of occurrence: 0.8 ), whereas the infrequent target stimulus was 2,000 Hz tone (duration: $200 \mathrm{~ms}$; rise-fall times: $10 \mathrm{~ms}$; intensity: $80 \mathrm{~dB}$ SPL; and probability of occurrence: 0.1 ). The novel sounds (probability of occurrence: 0.1 ) were unique, nonrepeating sound effects (novels) either sampled from a sound effects compact disk or generated in the laboratory using a microphone as recordings of typical environmental sounds (eg, a key in a lock and a cup being placed on a table). ${ }^{21,22}$ These novel sounds were clipped to a length of $200 \mathrm{~ms}$ and were unidentifiable and ambiguous. The intensities of all stimuli including novel sounds were checked using a calibrated sound-level meter (Radio Shack 33-2055) and adjusted so the perceived intensity by the subject was $80 \mathrm{~dB}$. The subjects were not informed that the novel sounds would be presented, and if they asked questions about their presence, they were reminded to count only to the target tones. The interstimulus interval varied randomly between 2 seconds and 3 seconds. The task lasted for $\sim 15$ minutes.

The ERPs were measured as grand averaged waveforms. Trials containing eye movements were automatically rejected. A further selection was performed in the offline analysis to reject other kinds of artifacts, according to the clinical guidelines. ${ }^{23}$ For each subject, all artifact-free trials were averaged per stimulus (target and novel) and filtered with a low-pass digital filter of $20 \mathrm{~Hz}$, for each stimulus. Scalp electrode activity was measured at all electrode sites 
Table I Mean scores of P3 components assessments at pretDCS and post-tDCS evaluations

\begin{tabular}{|c|c|c|c|c|c|}
\hline \multicolumn{3}{|c|}{ Event-related potential } & \multirow{2}{*}{$\begin{array}{l}\begin{array}{l}\text { Pre-tDCS } \\
\text { evaluation }\end{array} \\
384.8 \pm 48.4\end{array}$} & \multirow{2}{*}{$\begin{array}{l}\begin{array}{l}\text { Post-tDCS } \\
\text { evaluation }\end{array} \\
358.7 \pm 20.4\end{array}$} & \multirow{2}{*}{$\frac{P \text {-value }}{}$} \\
\hline Target & Lat (ms) P3b & $\mathrm{Fz}$ & & & \\
\hline & & $\mathrm{Cz}$ & $384.8 \pm 46.5$ & $361.3 \pm 20.3$ & $0.03^{*}$ \\
\hline & & $\mathrm{Pz}$ & $390.1 \pm 45.1$ & $366.3 \pm 28.5$ & $0.04 *$ \\
\hline & $\mathrm{Amp}(\mu \mathrm{V}) \mathrm{P} 3 \mathrm{~b}$ & $\mathrm{Fz}$ & $4.7 \pm 3.9$ & $6.5 \pm 6.1$ & 0.24 \\
\hline & & $\mathrm{Cz}$ & $6.4 \pm 4.5$ & $9.8 \pm 4.8$ & $0.02 *$ \\
\hline & & $\mathrm{Pz}$ & $9.2 \pm 6.1$ & $\mid I .1 \pm 5.9$ & 0.35 \\
\hline \multirow[t]{6}{*}{ Novel } & Lat (ms) P3a & $\mathrm{Fz}$ & $332.2 \pm 28.4$ & $316.4 \pm 35.7$ & 0.11 \\
\hline & & $\mathrm{Cz}$ & $329.4 \pm 32.7$ & $330.9 \pm 42.2$ & 0.88 \\
\hline & & $\mathrm{Pz}$ & $342.4 \pm 26.1$ & $336.4 \pm 46.7$ & 0.62 \\
\hline & $\mathrm{Amp}(\mu \mathrm{V}) \mathrm{P} 3 \mathrm{a}$ & $\mathrm{Fz}$ & $6.4 \pm 4.2$ & $4.7 \pm 4.3$ & 0.20 \\
\hline & & $\mathrm{Cz}$ & $8.5 \pm 4.5$ & $8.5 \pm 5.3$ & 0.98 \\
\hline & & $\mathrm{Pz}$ & $9.9 \pm 4.3$ & $9.9 \pm 4.7$ & 0.99 \\
\hline
\end{tabular}

Notes: $* P<0.05$. Data are presented as mean \pm standard deviation.

Abbreviations: tDCS, transcranial direct current stimulation; lat, latency; amp, amplitude.

of which $\mathrm{Fz}, \mathrm{Cz}$, and $\mathrm{Pz}$ were analyzed. $\mathrm{Fz}, \mathrm{Cz}$, and $\mathrm{Pz}$ were chosen for analyses because ERPs responses are largest on the midline locations.

The $\mathrm{P} 3 \mathrm{~b}$ and $\mathrm{P} 3 \mathrm{a}$ components were identified as the largest positive deflections between $250 \mathrm{~ms}$ and $500 \mathrm{~ms}$, respectively, in the target and novel responses. The amplitude of these components was identified by means of baseline to peak measurements. ${ }^{24}$

\section{Statistical analysis}

The Statistical Package for the Social Sciences (SPSS) was used for statistical calculations. All tests were two tailed with an alpha $=0.05$. Raw data were normally distributed and are reported in Table 1 as mean \pm standard deviation. Paired sample $t$-test was used to compare pretreatment and posttreatment measures.

\section{Results}

Two patients did not complete the stimulation protocol because of the onset of migraine. The remaining 25 patients tolerated tDCS without complications with the exception of three patients complaining of a transient burning sensation delimited to the electrode site. All patients correctly performed required tasks during each ERPs recording. Grand averaged waveforms for target stimulus at T0 and T1 are shown in Figure 1. Measurements of P3b components revealed a significant higher amplitude and shorter latency after tDCS treatment than baseline evaluation (latency: $\mathrm{Fz}$ $P=0.02, \mathrm{Cz} P=0.03$, and $\mathrm{Pz} P=0.04$; amplitude: $\mathrm{Fz} P=0.24$, $\mathrm{Cz} P=0.02$, and $\mathrm{Pz} P=0.35$; Table 1; Figure 1). No significant differences were observed in $\mathrm{P} 3 \mathrm{a}$ latency and amplitude in the two ERP sessions (Table 1).

\section{Discussion}

To the best of our knowledge, this is the first study investigating prefrontocerebellar tDCS potential in improving P300 performances on patients with euthymic BD. Our data showed that tDCS led to significant changes (larger amplitude and shorter latency) in the P3b parameters after stimulation, whereas the P3a component did not show any significant change.

The P300 is the most widely used and studied ERP component in psychiatry. ${ }^{25}$ This component reflects cognitive
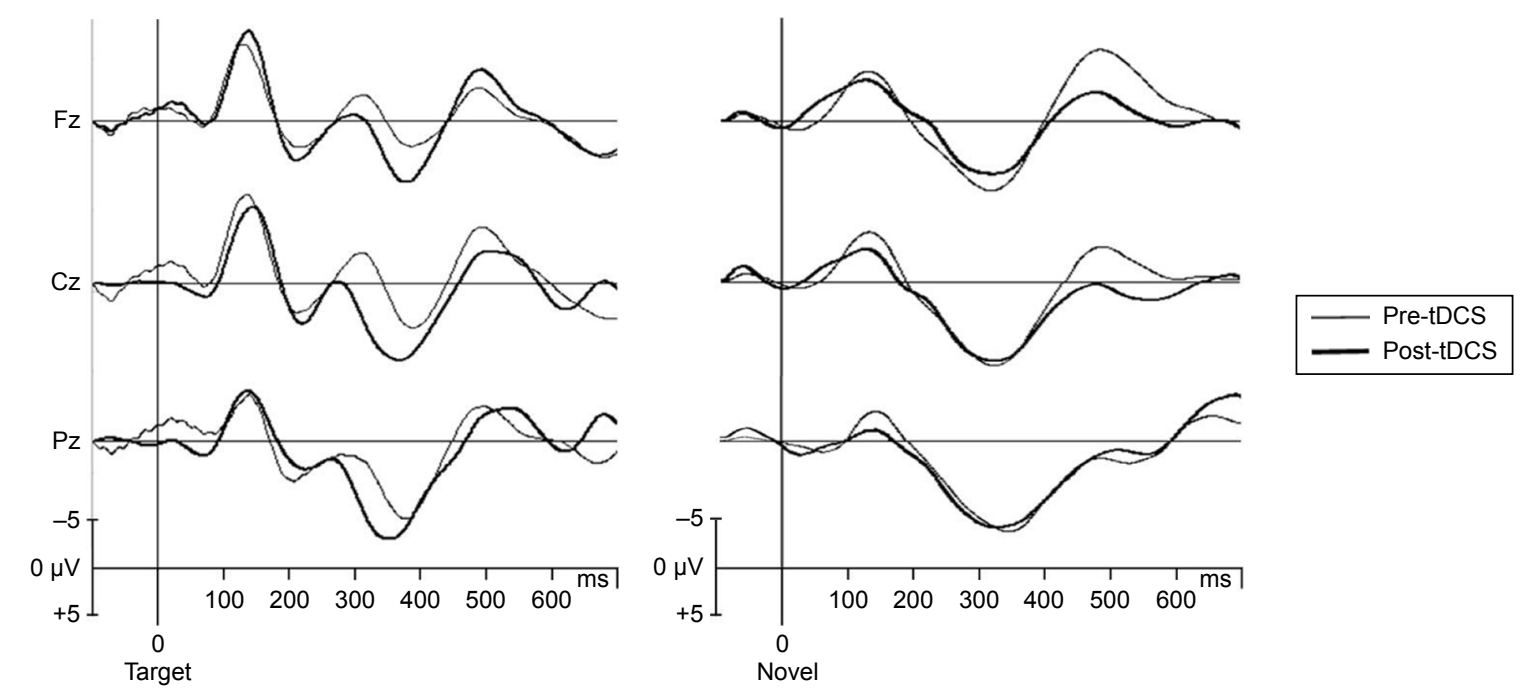

Figure I Grand averaged P3b and P3a traces at mid-line scalp sites at T0 (pre-tDCS: thin line) and TI (post-tDCS: thick line), respectively, for target and novel stimuli. Note: The analysis time was $800 \mathrm{~ms}$ with a $100 \mathrm{~ms}$ prestimulus baseline.

Abbreviation: tDCS, transcranial direct current stimulation. 
processes involved in stimulus categorization as an indicator of selective attention and memory updating. ${ }^{8-10}$ In particular, although the P3a, elicited by distracter stimulus, is related to an orienting response during a context-updating process, the $\mathrm{P} 3 \mathrm{~b}$ component is associated with task-relevant attentional mechanisms and decision-making processes. ${ }^{8}$ Our findings suggest an improvement in the context-updating function (as revealed by the significant changes in P3b), whereas the orienting phase of the stimulus processing was not significantly influenced by the treatment (as revealed by the nonsignificant changes in $\mathrm{P} 3 \mathrm{a}$ ).

The results of the present research preliminarily indicate that concomitant prefrontal excitatory and cerebellar inhibitory tDCS stimulation may have a positive effect on brain global information processing stream. It is thus possible to hypothesize that the observed functional improvements may be at least partially attributable to functional modulation of prefrontocerebellar circuitry activity, as suggested in the previous studies. ${ }^{11-16}$

From a clinical point of view, cognitive disturbances have gained considerable importance as critical features of psychiatric disorders, and it is now believed that they might represent valid therapeutic targets. ${ }^{9,26}$ It is currently accepted that cognitive impairment and symptomatic manifestations of mental disorders may require separate and integrated therapeutic approaches. ${ }^{9,26}$ As improvements in P300 performances have been associated with improvements in cognitive functioning, ${ }^{9}$ it might be hypothesized that the proposed prefrontocerebellar tDCS protocol could potentially be used as a complementary therapeutic approach with possible positive clinical outcomes.

The small sample size and the open-label design are major limitations of the study. Moreover, we assessed a relatively large number measures and we did not correct for multiple comparisons; therefore, these results should only be considered as preliminary/hypothesis generating. Among the strengths 1) this is one of the few studies using concomitant prefrontal-excitatory and cerebellar-inhibitory tDCS, as well as the first study to assess the effects of such stimulation through P300, 2) the sample was clinically well characterized, 3) P300 is a reliable marker of neurocognitive processes, and 4) during tDCS administration, patients' pharmacological maintenance therapies remained unchanged, allowing us to specifically account for tDCS effect on P300.

\section{Acknowledgment}

The research was fully funded by the Department of Neurology and Psychiatry of Sapienza University of Rome.

\section{Disclosure}

The authors report no conflicts of interest in this work.

\section{References}

1. Trede K, Salvatore P, Baethge C, Gerhard A, Maggini C, Baldessarini RJ. Manic-depressive illness: evolution in Kraepelin's textbook, 1883-1926. Harv Rev Psychiatry. 2005;13(3):155-178.

2. Kessler RC, Akiskal HS, Ames M, et al. Prevalence and effects of mood disorders on work performance in a nationally representative sample of U.S. workers. Am J Psychiatry. 2006;163(9):1561-1568.

3. Kogan JN, Otto MW, Bauer MS, et al. Demographic and diagnostic characteristics of the first 1,000 patients enrolled in the Systematic Treatment Enhancement Program for Bipolar Disorder (STEP-BD). Bipolar Disord. 2004;6(6):460-469.

4. Tohen M, Zarate CA Jr, Hennen J, et al. The McLean-Harvard FirstEpisode Mania Study: prediction of recovery and first recurrence. $\mathrm{Am}$ J Psychiatry. 2003;160(12):2099-2107.

5. Morris CD, Miklowitz DJ, Wisniewski SR, Giese AA, Thomas MR, Allen MH. Care satisfaction, hope, and life functioning among adults with bipolar disorder: data from the first 1,000 participants in the Systematic Treatment Enhancement Program. Compr Psychiatry. 2005; 46(2):98-104.

6. Bersani G, Polli E, Valeriani G, et al. Facial expression in patients with bipolar disorder and schizophrenia in response to emotional stimuli: a partially shared cognitive and social deficit of the two disorders. Neuropsychiatr Dis Treat. 2013;9:1137-1144.

7. Martínez-Arán A, Vieta E, Colom F, et al. Cognitive impairment in euthymic bipolar patients: implications for clinical and functional outcome. Bipolar Disord. 2004;6(3):224-232.

8. Polich J. Updating P300: an integrative theory of P3a and P3b. Clin Neurophysiol. 2007;118(10):2128-2148.

9. Campanella S. Why it is time to develop the use of cognitive eventrelated potentials in the treatment of psychiatric diseases. Neuropsychiatr Dis Treat. 2013;9:1835-1845.

10. Huang WJ, Chen WW, Zhang X. The neurophysiology of P $300-$ an integrated review. Eur Rev Med Pharmacol Sci. 2015;19(8):1480-1488.

11. Benson BE, Willis MW, Ketter TA, et al. Interregional cerebral metabolic associativity during a continuous performance task (part II): differential alterations in bipolar and unipolar disorders. Psychiatry Res. 2008;164(1):30-47.

12. Kimbrell TA, Ketter TA, George MS, et al. Regional cerebral glucose utilization in patients with a range of severities of unipolar depression. Biol Psychiatry. 2002;51(3):237-252.

13. Minichino A, Bersani FS, Bernabei L, et al. Prefronto-cerebellar transcranial direct current stimulation improves visuo-spatial memory, executive functions and neurological soft signs in patients with euthymic bipolar disorder. Neuropsychiatr Dis Treat. 2015;11:2265-2270.

14. Minichino A, Bersani FS, Spagnoli F, et al. Prefronto-cerebellar transcranial direct current stimulation improves sleep quality in euthymic bipolar patients: a brief report. Behav Neurol. 2014;2014:876521.

15. Minichino A, Bersani FS, Trabucchi G, et al. The role of cerebellum in unipolar and bipolar depression: a review of the main neurobiological findings. Riv Psichiatr. 2014;49(3):124-131.

16. Delle Chiaie R, Minichino A, Salviati M, et al. Bipolar spectrum disorders in patients with cerebellar lesions: a comparison with Parkinson's disease. J Nerv Ment Dis. 2015;203(9):725-729.

17. Bersani FS, Biondi M. Historical recurrences in psychiatry: somatic therapies. Riv Psichiatr. 2012;47(1):1-4.

18. Poreisz C, Boros K, Antal A, Paulus W. Safety aspects of transcranial direct current stimulation concerning healthy subjects and patients. Brain Res Bull. 2007;72(4-6):208-214.

19. First MB. Structured Clinical Interview for DSM-IV Axis I Disorders. Washington, DC: American Psychiatric Press; 1997.

20. Biondi M, Bersani FS, Valentini M. The Italian edition of DSM-5. Riv Psichiatr. 2014;49(2):57-60. 
21. Bersani FS, Minichino A, Fattapposta F, et al. P300 component in euthymic patients with bipolar disorder type I, bipolar disorder type II and healthy controls: a preliminary event-related potential study. Neuroreport. 2015;26(4):206-210.

22. Kimble M, Kaloupek D, Kaufman M, Deldin P. Stimulus novelty differentially affects attentional allocation in PTSD. Biol Psychiatry. 2000;47(10):880-890.

23. Duncan CC, Barry RJ, Connolly JF, et al. Event-related potentials in clinical research: guidelines for eliciting, recording, and quantifying mismatch negativity, P300, and N400. Clin Neurophysiol. 2009;120(11): 1883-1908.
24. Picton TW, Bentin S, Berg P, et al. Guidelines for using human eventrelated potentials to study cognition: recording standards and publication criteria. Psychophysiology. 2000;37(2):127-152.

25. Thaker GK. Neurophysiological endophenotypes across bipolar and schizophrenia psychosis. Schizophr Bull. 2008;34(4):760-773.

26. Harvey PD, Bowie CR. Cognitive remediation in severe mental illness. Innov Clin Neurosci. 2012;9(4):27-30.

\section{Publish your work in this journal}

Neuropsychiatric Disease and Treatment is an international, peerreviewed journal of clinical therapeutics and pharmacology focusing on concise rapid reporting of clinical or pre-clinical studies on a range of neuropsychiatric and neurological disorders. This journal is indexed on PubMed Central, the 'PsycINFO' database and CAS, and is the official journal of The International Neuropsychiatric Association (INA). The manuscript management system is completely online and includes a very quick and fair peer-review system, which is all easy to use. Visit http://www.dovepress.com/testimonials.php to read real quotes from published authors.

Submit your manuscript here: http://www.dovepress.com/neuropsychiatric-disease-and-treatment-journal 